Mycobacterium tuberculosis ${ }^{4}$, possibly explaining the well documented reports that vitamin $D$ therapy cures chronic skin tuberculosis ${ }^{5}$. Because T lymphocytes also contain the receptor, presumably they are also affected by the metabolite, but with what functional significance is not clear ${ }^{6}$.

Thus vitamin $\mathrm{D}_{3}$ metabolites have immunologically relevant effects on the lymphoid system, and their levels within this system are locally regulated by $\mathrm{T}$ cells, gamma interferon and macrophages, in a manner which may usually be quite independent of the regulation of systemic levels. When interferon-induced macrophage activation is intense, however, 'overspill' of the 1,25-dihydroxy metabolite can occur, causing sufficient rises in systemic levels to induce hypercalcaemia, as often seen in sarcoidosis ${ }^{2}$, and sometimes in tuberculosis?

Once again the macrophage proves to be a cell with an amazing range of potential functions.

G.A.W. RoOK J. STEELE

Department of Microbiology,

Middlesex Hospital Medical School, Riding House Street, London WIP 7PP, $U K$

1. Dickson, I. Nature 325, 18 (1987)

2. Adams, J.S. \& Gacad, M.A. J. exp. Med. 90, 755-765 (1985).

3. Koeffler, H.P., Reichel, H., Bishop, J.E. \& Norman, A.W Biochem. biophys. Res. Comm. 127, 596-603 (1985).

4. Rook, G.A.W. et al. Immunology 57, 159-163 (1986).

5. Dowling, G.S. \& Prosser Thomas, E.W. Br. J. Derm. 58, 45-52 (1946)

6. Provvedini, D.M. et al. Science 221, 1181-1183 (1983).

7. Abassi, A.A. et al. Ann. intern. Med. 90, 324-328 (1979).

\section{Models for the origin of influenza viruses}

SIR-Hoyle and Wickramasinghe have recently repeated $^{1.2}$ their hypothesis ${ }^{3}$ that the Earth is continually bombarded by influenza viruses from comets. Their 'comet' model denies transmission of influenza viruses between individuals, each infection being contracted directly from virus particles falling through the upper atmosphere. The time is past for such qualitative speculations now that the predictions from the comet model can be falsified by quantitative tests.

The predictions concern the minimallength trees that can be constructed on the basis of nucleic-acid sequences. The largest set of sequences for one strain of influenza virus from the same host are nine haemagglutinin sequences covering the years 1933 to 1980 of the human $\mathrm{H} 1$ subtype. The simplest comet model is that the different isolates of $\mathrm{H} 1$ influenza are carried on different comets, having developed independently from a common ancestor that existed at some distant time in the past. On this model the sequences would appear generally similar because of their common origin (thus explaining their homology), but the differences between
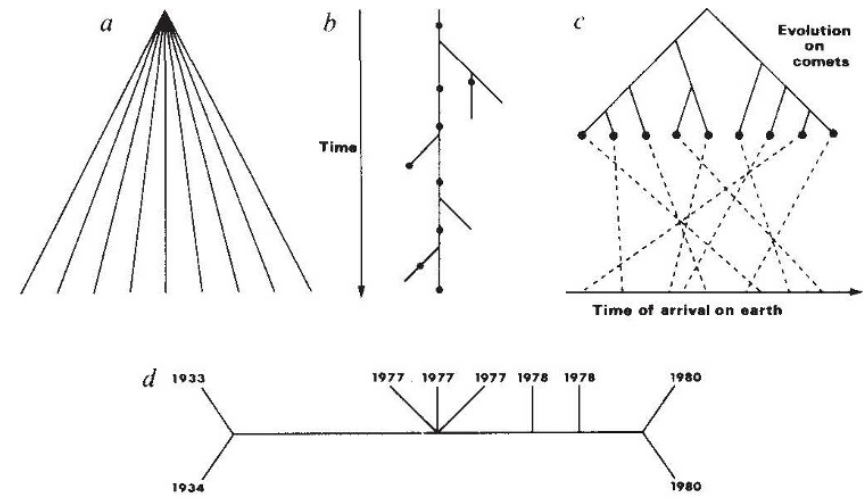

Fig. 1 Trees representing different models. $a$, The Big-Bang or star tree model. $b$, The biological model (for example ref. 7). $c$, Modified comet model (independent arrival). $d$, The minimal tree $^{8}$ for the nine viral sequences from the EMBL database ${ }^{9}$, edges of zero length are contracted.

different pairs of taxa would be independent. This is the Big-Bang model ${ }^{4}$, and is represented by the star tree $e^{4.5}$ shown in Fig. 1a. If, for example, three viral isolates had adenine at one nucleotide position and the other six had cytosine then the star tree would require three nucleotide changes while the biological model, which assumes that viral strains share common ancestors (Fig. 1b), could require one, two or three changes. The expected number of nucleotide changes for a minimallength tree, given the Big-Bang model, can be calculated (unpublished data). For these nine sequences, the expected number is 163 whereas the observed number is 95. This has a probability of occurring of about $10^{-58}$, assuming that the viruses arose independently from a common ancestor. Thus we reject the star tree model.

The observed number of changes is however consistent with an evolutionary model whether it be terrestrial or extraterrestrial. For example the second version of the comet model (Fig. 1c) assumes that viruses on different comets are related through some ancient extraterrestrial evolutionary process but that they arrive independently on earth from different comets. Viruses on two comets may share a more recent ancestor than with those on a third comet but any relationships among the viruses should show no significant correlation with their order of arrival on earth. This leads to the prediction that influenza viruses isolated in consecutive years should not be more closely related than those isolated several years apart. In fact, the viruses on the minimal tree (Fig. $1 d$ ) are in chronological sequence, consistent with the biological model. There is one chance in 7,560 $(=9 ! / 8.6 .2)$ of obtaining this time sequence if the viruses on the minimal tree arrived independently in time. Extending the data set to 12 viral isolates (1933-1982) for which a smaller part (312 nucleotides) of the haemagglutinin gene is sequenced, leads to an even smaller probability. Twelve taxa with independent arrival times, will produce a chronological order as good as that observed (one pair of adjacent isolates interchanged) about once in 1,500,000 trials. Consequently we reject this second comet model.

Models invoking only a single comet do not fit the data either. One such possibility is that of a single comet carrying an evolving virus that periodically infects the Earth as the comet passes it. But the pattern in the arrival of virus isolates, the short times between altered sequences, the lack of a slow mixing compartment in the atmosphere and the lack of randomization of sequences to give a "quasi-species' ${ }^{16}$ lead us to conclude that this single-comet hypothesis cannot explain the evolution of influenza viruses while still retaining the assumption of non-transmission between host individuals.

I.M. HENDERSON DAVID PENNY

Department of Botany and Zoology,

Michael D. Hendy

Department of Mathematics and Statistics, Massey University,

Palmerston North, New Zealand

1. Hoyle, F. \& Wickramasinghe, C. Nature 322, 509-51 (1986)

2. Hoyle, F. \& Wickramasinghe, C. Living Comets (University Cardiff College Press, 1984).

Hoyle, F. \& Wickramasinghe, C. Diseases from Space (Dent, London, 1979)

4. Thompson, E.A. Human Evolutionary Trees (Cambridge University Press, 1975).

. Eigen, M. \& Winkler-Oswatatisch, R. Naturwissenschaften 68, 217-228 (1981)

6. Eigen, M. \& Schuster, P. Naturwissenschaften 65, 341-369 (1978)

7. Raymond, F.L. et al. Virology 148, 275-287 (1986).

8. Hendy, M.D. \& Penny, D. Math. Biosc. 59, 277-290(1982)

9. Hamm, G.H.\& Cameron, G.N. Nucleic Acid Res. 14, 5-10 (1986).

\section{Scientific Correspondence}

Scientific Correspondence is intended to provide a forum in which readers may raise points of a scientific character. They need not arise out of anything published in Nature. In any case, priority will be given to letters of less than 500 words and five references. 